\title{
Inhibition of p21-activated kinase 4 expression suppresses the proliferation of Hep-2 laryngeal carcinoma cells via activation of the ATM/Chk1/2/p53 pathway
}

\author{
XIN SUN, BIN LIU, JIN WANG, JUN LI and WEN-YUE JI
}

Department of Otorhinolaryngology, Shengjing Hospital, China Medical University, Shenyang, Liaoning 110001, P.R. China

Received September 25, 2012; Accepted November 12, 2012

DOI: $10.3892 /$ ijo.2012.1718

\begin{abstract}
In the present study, we investigated the effects of the p21-activated kinase 4 (Pak4) gene on Hep-2 laryngeal carcinoma cells in vivo and in vitro. The expression of Pak4 was downregulated using small interfering RNA (siRNA). The downregulation of Pak4 decreased the proliferation and increased apoptosis and $\mathrm{S}$ phase arrest in Hep-2 cells in vitro. In further experiments, we determined that the $S / G_{2}$ transition was obstructed by the downregulation of Pak4 using 5-chloro2'-deoxyuridine (CldU) and 5-iodo-2'-deoxyuridine (IdU) double staining. A xenografted Hep-2 tumor mouse model was created by inducing human tumors with a subcutaneous (s.c.) injection of $5 \times 10^{6} \mathrm{Hep}-2$ cells into the dorsal flank region of nu/nu mice. The downregulation of Pak4 in established xenografted tumors decreased tumor size and weight. The survival rate of the mice with tumors that did not express Pak4 was significantly higher compared to the mice with tumors expressing Pak4. These results confirm the role of Pak4 as an oncogene in laryngeal carcinoma cells. To identify the mechanism of the cell cycle arrest induced by Pak4, immunohistochemical staining was performed to detect changes in cell cycle-related proteins. The results demonstrated that p53 was activated following the downregulation of Pak4. The levels of ataxia telangiectasia mutated (ATM), the upstream protein of checkpoint kinase (Chk)1 and Chk2, also increased. Therefore, we confirmed that the mechanisms of the Pak4induced cell cycle arrest invovlve the activation of the ATM/ Chk1/2/p53 pathway. These results may prove helpful for the development of novel therapies for the treatment of laryngeal carcinoma.
\end{abstract}

Correspondence to: Dr Wen-yue Ji, Department of Otorhinolaryngology, Shengjing Hospital, China Medical University, 36 Sanhao Street, Shenyang, Liaoning 110004, P.R. China

E-mail: ji_wenyue2004@163.com

Key words: laryngeal carcinoma, p21-activated kinase 4, cell cycle arrest, survival rate, CldU and IdU double staining

\section{Introduction}

The Rho GTPases, such as Cdc42, Rac and Rho, can impact cell morphology and migration by regulating the actin cytoskeleton (1). The Rho GTPases can also regulate proliferation and activate signaling pathways, including the JNK/MAPK and JNK/ SAPK signaling pathways (2-6). The Rho GTPases are required for binding to certain proteins (1). The p21-activated kinase (Pak) family of serine/threonine kinases are major target proteins of the Rho GTPases (7). Six mammalian Pak proteins have been identified in this family and have been classified into 2 groups: group 1 Paks (Pak1-Pak3) and group 2 Paks (Pak4-Pak6) (8). Pak4 was first identified as an effector of Cdc42 in mouse embryonic fibroblast cell lines (9). The overexpression of Pak4 has been observed in a number of cancer cell lines (10). Pak4 has also been shown to be overexpressed in many types of cancer, such as esophageal squamous cell carcinoma (11) and mouse colon tumors (12). However, to our knowledge, little is known about the individual role of Pak4 in Hep-2 laryngeal carcinoma cells.

Laryngeal squamous cell carcinoma (LSCC) constitutes almost 2 to $3 \%$ of all malignant tumors, with a total of 159,000 new cases of carcinoma per year, more commonly affecting males (13). In China, diagnostic and therapeutic modalities for laryngocarcinoma have improved over the past few years. However, the incidence of LSCC is gradually rising, particularly in Northeastern China (14).

In the present study, we investigated the effects of Pak4 on Hep-2 laryngeal carcinoma cells by regulating its expression in vitro and in vivo. The mechanisms of the effect of Pak4 on laryngeal carcinoma cells were also investigated.

\section{Materials and methods}

Cell lines and cell culture. Hep-2 laryngeal carcinoma cells were obtained from the American Type Culture Collection (ATCC; Bethesda, MD) and maintained in RPMI-1640 medium (Life Technologies, Inc., Gaithersburg, MD) supplemented with $10 \%(\mathrm{v} / \mathrm{v})$ fetal bovine serum (FBS) and antibiotics (100 units $/ \mathrm{ml}$ of penicillin and $100 \mathrm{mg} / \mathrm{ml}$ of streptomycin) at $37^{\circ} \mathrm{C}$ in a $5 \%(\mathrm{v} / \mathrm{v}) \mathrm{CO}_{2}$ incubator.

siRNA against Pak4 and transfection. Pak4 small interfering RNA (siRNA) oligonucleotides were purchased from 
Dharmacon, Inc. (Lafayette, CO). Cells were seeded onto 60-mm plates for $24 \mathrm{~h}$ and transfected with siRNA or control siRNA for $48 \mathrm{~h}$ using Lipofectamine 2000 (Invitrogen Life Technologies, Carlsbad, CA) according to the manufacturer's instructions.

$R T$-PCR. Total RNA was isolated using an RNeasy Mini kit (BioMed, Beijing, China). cDNA was reverse transcribed with $1 \mu \mathrm{g}$ of total RNA, using the Takara Reverse Transcription kit (Takara Dalian, Dalian, China). cDNA was amplified using the following primers: the sequences of the forward and reverse primers for $P a k 4$ were 5'-GACATCAAGAGCGACTCGATCC-3' and 5'-ATCACCATTATCCCCAGCGAC-3', respectively. $G A P D H$ was used as the internal control. The sequences of the forward and reverse primers for $G A P D H$ were 5'-AGAAGGC TGGGGCTCATTTG-3' and 5'-AGGGGCCATCCACAG TCTTC-3', respectively. PCR was performed for 35 cycles under the following conditions: annealing at $56^{\circ} \mathrm{C}(15 \mathrm{sec})$, extension at $72^{\circ} \mathrm{C}(30 \mathrm{sec})$ and denaturing at $94^{\circ} \mathrm{C}(30 \mathrm{sec})$ using a Takara thermal cycler.

Western blot analysis. Total cell extracts were obtained using lysis buffer containing $20 \mathrm{mM}$ HEPES (pH 7.4), $150 \mathrm{mM} \mathrm{NaCl}$, $1 \mathrm{mM} \mathrm{MgCl}_{2}, 1 \mathrm{mM}$ EDTA, $10 \%$ glycerol, $1 \%$ Triton $\mathrm{X}-100$, $1 \mu \mathrm{g} / \mathrm{ml}$ leupeptin and $1 \mu \mathrm{g} / \mathrm{ml}$ aprotinin. Equal amounts $(90 \mu \mathrm{g})$ of cell lysates were separated by $10 \%$ SDS-polyacrylamide gel electrophoresis, transferred onto polyvinylidene difluoride membranes and incubated with the following specific antibodies: Pak4 antibody (Abcam PLC, Cambridge, UK) was used to identify transfection efficiency. $\beta$-actin (Santa Cruz Biotechnology, Inc., Santa Cruz, CA) was used as the internal control. The reaction was followed by probing with peroxidase-coupled secondary antibodies including anti-rabbit IgG, or anti-mouse IgG antibodies at dilutions ranging from 1:1,000 to 1:2,000 (Amersham Biosciences, Needham, MA) and binding results were visualized by enhanced chemiluminescence (Amersham Pharmacia Biotech, Piscataway, NJ).

Colony formation assay. For the colony formation assay, cells (200 cells per well) were seeded in 24-well tissue culture plates. Plates were incubated for 3 weeks in a humidified incubator at $37^{\circ} \mathrm{C}$. Three weeks after seeding, colonies were stained with $0.05 \%$ crystal violet containing $50 \%$ methanol and counted. The colonies were counted in 4 to 5 random fields from each of the duplicate samples by using a microscope at x100 magnification.

Measurement of caspase-3 and -9 activities. Caspase activities were measured by colorimetric assay kits (KeyGen Biotech. Co., Ltd., Nanjing, China) according to the manufacturer's instructions. After harvesting, cells were washed in ice-cold PBS and lysed; proteins were extracted and stored at $-80^{\circ} \mathrm{C}$. Cell lysates $(20 \mu \mathrm{l})$ were then added to a buffer containing a p-nitroaniline (pNA)-conjugated substrate for caspase-3 (Ac-DEVD-pNA), or -9 (LEHD-pNA) to a total of $100 \mu 1$ reaction volume. Incubation was carried out at room temperature for caspase-3 and -9 followed by shaking at $500 \mathrm{rpm}$ for $1 \mathrm{~min}$ and then incubation at room temperature for $2 \mathrm{~h}$. The concentration of the released pNA in each well was measured using a plate-reading luminometer (Thermo Fisher Scientific, Beijing, China). Data were from 3 independent experiments.
Cell cycle analysis. The Hep-2 laryngeal carcinoma cells (3x10 $/$ well) were plated and incubated overnight. The control and treated cells were trypsinized, collected in PBS and fixed on ice with $1 \%$ paraformaldehyde, followed by $70 \%$ cold ethanol. Following treatment with $10 \mu \mathrm{g} / \mathrm{ml}$ RNase, the cells were stained with $50 \mu \mathrm{g} / \mathrm{ml}$ propidium iodide (PI; KeyGen Biotech. Co., Ltd.) for $15 \mathrm{~min}$ at room temperature for cell cycle analysis. The stained cells were analyzed by flow cytometry. Data analysis was performed with CellQuest software (BD Biosciences, Rockville, MD).

Labeling of cells with thymidine analogs. Actively replicating cells at the beginning of each hour of the $S$ phase were first labeled with the thymidine analog, 5-iodo-2'-deoxyuridine (IdU; $50 \mu \mathrm{M}$ ) (Sigma-Aldrich, Carlsbad, CA) for $40 \mathrm{~min}$, washed 3 times with PBS and then labeled with 5-chloro-2'deoxyuridine (CldU; $100 \mu \mathrm{M}$ ) (Sigma) for $40 \mathrm{~min}$. IdU and CldU incorporated into replicating DNA were later detected with red or green fluorescent antibodies, respectively, as described below. Three independent replicate experiments were performed and analyzed for the control, mock and treated cells.

Immunostaining. Slides were treated with $70 \%$ ethanol, washed in PBS, denatured in $2.5 \mathrm{M} \mathrm{HCl}$ for $30 \mathrm{~min}$ and permeabilized in $0.25 \%$ Triton X-100 for 5 min and blocked with $1 \%$ bovine serum albumin. The slides were incubated at room temperature with the following antibodies: i) 1:500 mouse anti-bromodeoxyuridine (detects IdU) (Sigma); ii) 1:1,000 Alexafluor 488-conjugated anti-mouse (Sigma); iii) 1:2,000 rat anti-bromodeoxyuridine (detects CldU) (Santa Cruz Biotechnology, Inc.); and iv) 1:1,000 Alexafluor 633-conjugated anti-rat antibodies (Invitrogen Life Technologies).

After counterstaining with DAPI $(1 \mu \mathrm{g} / \mathrm{ml})$ (KeyGen Biotech. Co., Ltd.), photographic images were taken using an Olympus CX71 fluorescence microscope (Olympus, Tokyo, Japan).

Xenografted Hep-2 tumor mouse model. All in vivo experiments were approved by the Ethics Committee of China Medical University. Human tumors were induced by a subcutaneous (s.c.) injection of $5 \times 10^{6} \mathrm{Hep}-2$ cells into the dorsal flank region of nu/nu mice (CAnN.Cg-Foxn1 ${ }^{\text {nu}} / \mathrm{Crl}$; Vital River, Beijing, China). When tumor volumes had reached a mean volume of $50 \pm 5 \mathrm{~mm}^{3}$, the animals were randomized into 3 groups $(n=15$ per group). All mice received 2 injections, $72 \mathrm{~h}$ apart (days 1 and 4). Injections were administered by mixing siRNA $(20 \mu \mathrm{g})$ with Lipofectamine $2000(30 \mu \mathrm{l})$ and PBS in a total volume of $100 \mu \mathrm{l}$. Tumors were measured with calipers every 5 days and tumor volumes were calculated (tumor volume $=$ length $\mathrm{x}$ width ${ }^{2}$ x 0.52) (15).

An additional 180 mice were used to establish xenografts to observe survival time. For these experiments, mice with xenograft tumors were treated as described above. Survival was monitored until the experiments were terminated due to heavy tumor burden.

Immunohistochemical staining. Immunohistochemical staining was performed on 4- $\mu \mathrm{m}$ sections obtained from formalin-fixed, paraffin-embedded blocks. Endogenous peroxidase activity was blocked with $3 \%$ hydrogen peroxide for $30 \mathrm{~min}$. Antigen 
retrieval was carried out in citrate buffer $(10 \mathrm{mM}, \mathrm{pH} 6.0)$ for $30 \mathrm{~min}$ at $95^{\circ} \mathrm{C}$ in a microwave oven (16). Sections were incubated with primary antibody at $4^{\circ} \mathrm{C}$ overnight. Cell cycle checkpoint-regulated proteins were probed with: anti-ataxia telangiectasia mutated (ATM), anti-p53, anti-checkpoint kinase (Chk)1, anti-Chk2 (Santa Cruz Biotechnology, Inc.), anti-phospho-S345-Chk1 and anti-phospho-T68-Chk2 antibodies (Cell Signaling Technology, Danvers, MA). Cell cycle-regulated proteins were probed with: anti-Cyclin A (Santa Cruz Biotechnology, Inc.) and anti-cyclin-dependent kinase 2 (CDK2) antibodies (Abcam PLC). The sections were then incubated with a biotinylated secondary antibody and exposed to a streptavidin complex (HRP). Positive reactions were visualized with 3,3'-diaminobenzidine tetrahydrochloride (DAB; Sigma), followed by counterstaining with hematoxylin. Sections treated without primary antibodies were used as negative controls.

Statistical analysis. Data were analyzed using GraphPad Prism 5 software. Statistical analysis was performed using a one-tailed Student's t-test (unilateral and unpaired). Kaplan-Meier survival plots were generated and comparisons between survival curves were made using log-rank statistical analysis. P-values $<0.05$ were considered to indicate statistically significant differences.

\section{Results}

mRNA and protein levels of Pak4 were evaluated in Hep-2 laryngeal carcinoma cells. The levels of Pak4 in siRNAtreated Hep-2, untreated and mock-treated cells were detected using western blot and RT-PCR assays. In the western blot assays, the levels of Pak4 protein were higher in the untreated Hep-2 and mock-treated Hep-2 cells compared with the siRNA-treated Hep-2 cells (Fig. 1, upper panel). To determine the correlation between the levels of Pak4 and the transcription of Pak4, RT-PCR analysis of Pak4 was performed. In these assays, the levels of Pak4 mRNA were higher in the untreated Hep-2 cells compared to the treated ones and were consistent with the levels of Pak4 detected by western blot analysis (Fig. 1, lower panel).

Pak4 is required for tumor growth in Hep-2 cells. Pak4 mRNA and protein levels are high in Hep-2 cells. We determined whether its downregulation would reduce the tumorigenicity of laryngeal carcinoma cells. Clonogenic assay showed that the proliferation rate of siRNA-treated Hep-2 cells was decreased compared to the untreated and mock-treated cells (Fig. 2A, $\mathrm{p}<0.05)$. Caspase -3 and caspase -9 activities in the treated cells was also found to be higher compared to the untreated and mock-treated cells (Fig. 2B, p<0.05). PI staining of cells revealed that Pak4-siRNA cells were arrested in the $S$ phase (Fig. 3, p<0.05).

Hep-2 cells were obstructed in the $S / G_{2}$ transition following siRNA treatment. To further analyze the proliferation activity of Hep-2 cells after siRNA treatment, a CldU/IdU doublelabeling method was applied to distinguish CldU and IdU incorporated into cellular DNA. According to the method of Bakker et al (17), we assessed the $G_{1} / S$ and $S / G_{2}$ checkpoint in Hep-2 cells following siRNA treatment. Our labeling strategy is shown in Fig. 4 (upper panel). In this assay, single-labeled

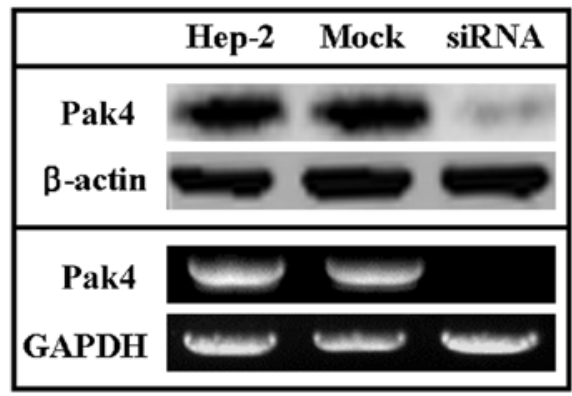

Figure 1. The levels of Pak4 protein and mRNA were measured in the Hep-2 laryngeal carcinoma cell line. (A) The expression of Pak4 protein was determined by western blot analysis of Hep-2 cells, Hep-2 cells treated with control siRNA (Mock cells) and Hep- 2 cells treated with siRNA. $\beta$-actin expression was used as the internal control (upper panel). (B) The mRNA level of Pak4 was determined by RT-PCR; GAPDH expression was used as the internal control (lower panel). Each experiment was performed in triplicate.
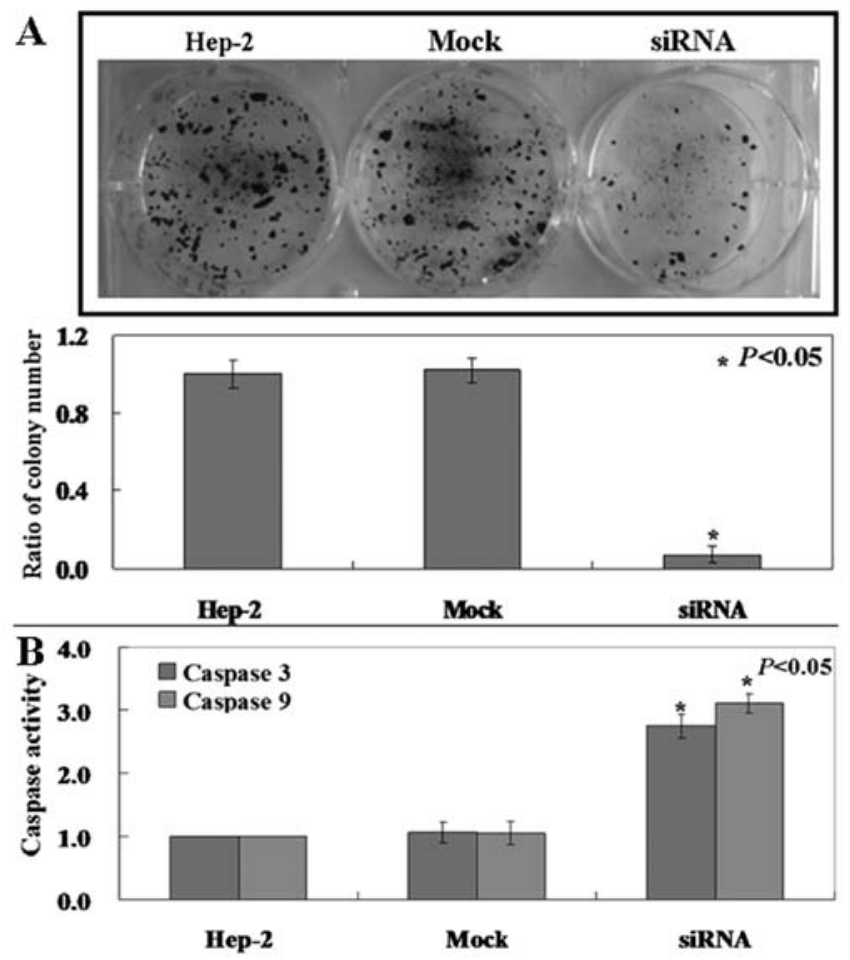

Figure 2. The proliferation rate of Pak4-siRNA-treated cells. (A) The percentages of growth inhibition of the downregulation of Pak4 in Hep-2 cells were determined by colony formation assay in comparison with the untreated cells $(\mathrm{p}<0.05)$. (B) Caspase assays confirmed the involvement of the intrinsic apoptotic pathway. The significant activation of caspase- 9 and caspase- 3 was observed $(\mathrm{p}<0.05)$.

IdU cells (green) are cells that enter the $\mathrm{S}$ phase after the first labeling period of the experiment. Single-labeled CldU cells (red) are cells that are left int he $\mathrm{S}$ phase before the second labeling period of the experiment. Cells labeled both with CldU and IdU (yellow) are in the S phase. Non-labeled cells are not in the $\mathrm{S}$ phase. This method helped us to identify the percentage of cells in the $G_{1} / S$ and $S / G_{2}$ transitions. As shown by our results, there was no difference in the number of Hep- 2 cells and mock cells in the $G_{1} / S$ transition, $S$ phase 

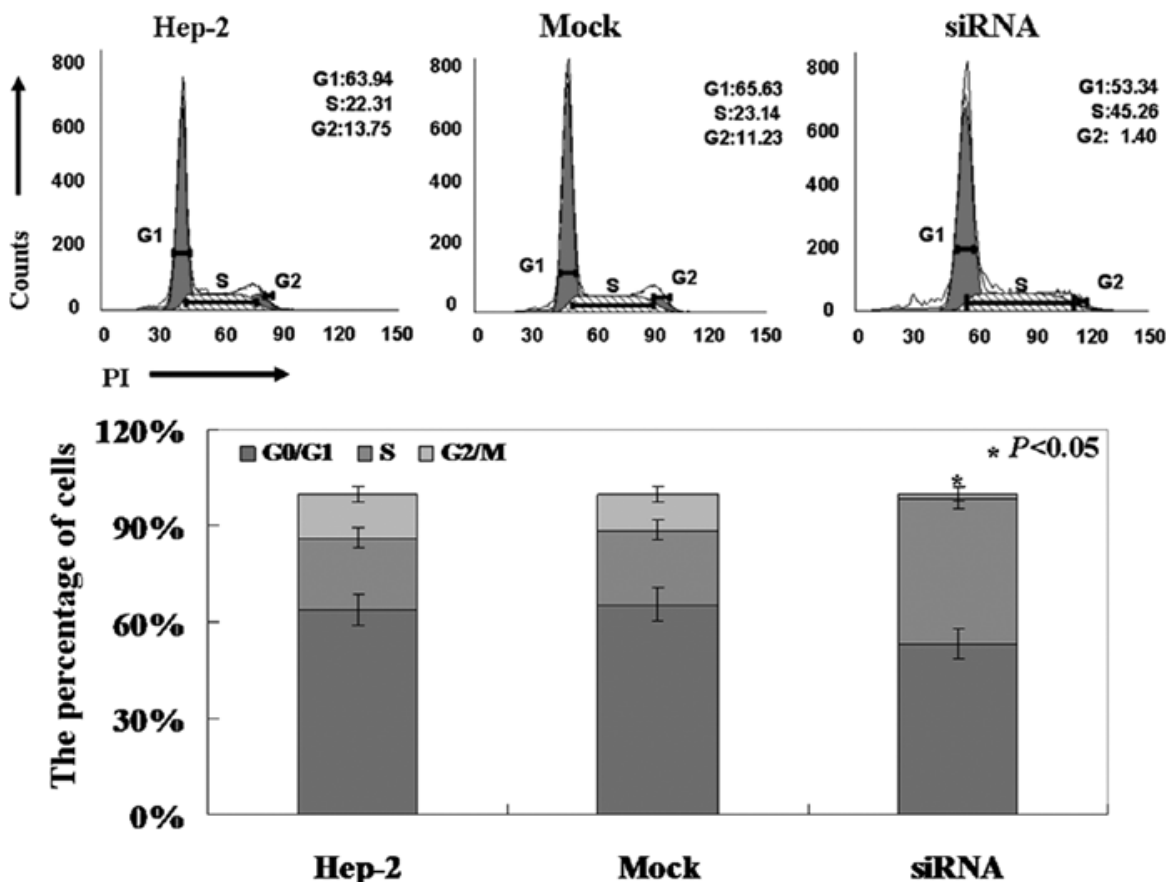

Figure 3. Change in the cell cycle were determined by PI staining. Treatment with Pak4-siRNA led to cells being arrested in the S phase. The histogram indicates that the results had statistical significance $(\mathrm{p}<0.05)$.

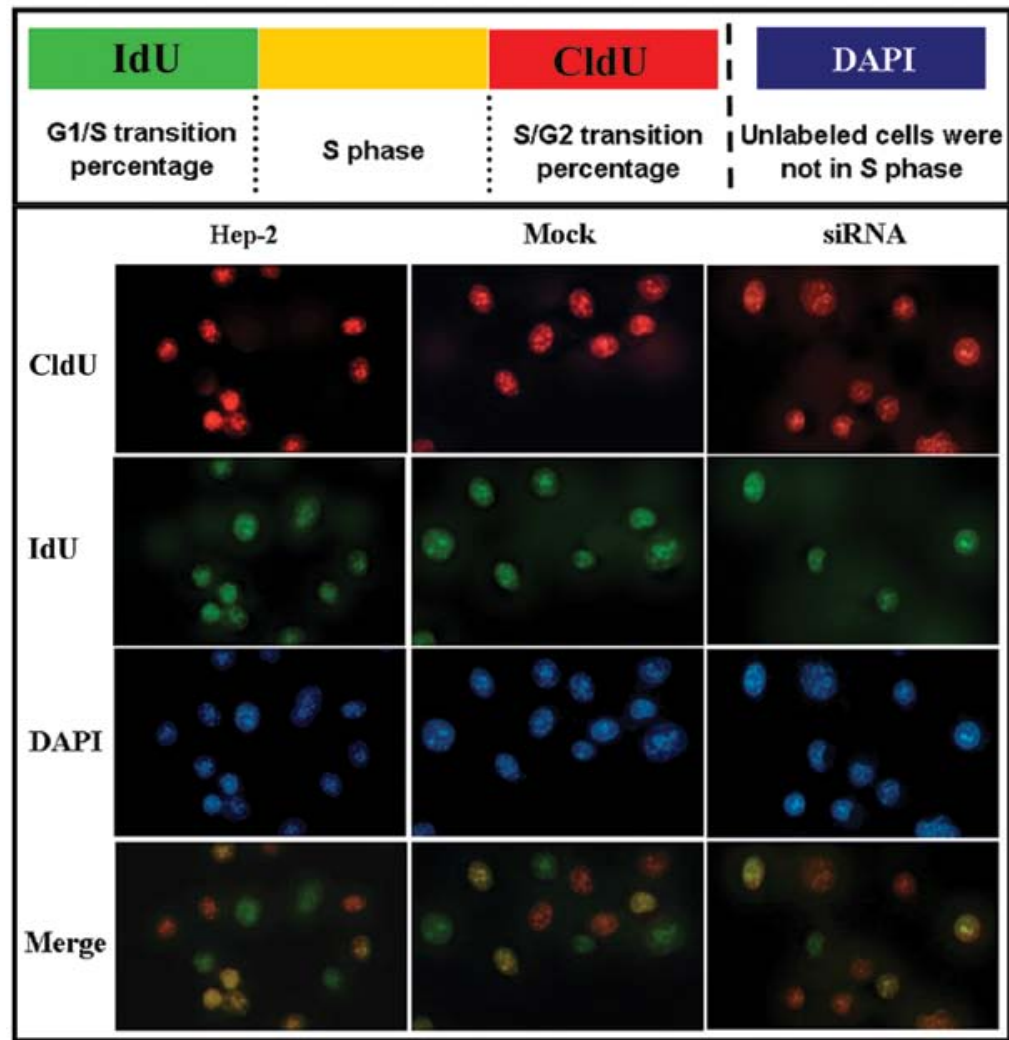

Figure 4. Downregulation of Pak4 induced the $S / G_{2}$ transition arrest. A diagram of our strategy used to assess the $S / G_{2}$ checkpoint in Hep-2 cells (upper panel). High magnification images captured using the $\mathrm{x} 40$ objective of Hep-2 cells, Mock cells and Hep-2 cells treated with siRNA. Representative images showing cells labeled with only IdU (Alexafluor 488), only CldU (Alexafluor 633), both IdU and CldU, or neither replication label (lower panel).

and $\mathrm{S} / \mathrm{G}_{2}$ transition. Non-labeled cells were considered cells in other phases (Fig. 4, lower panel). However, we found that the number of Hep-2 cells following siRNA treatment in the
$\mathrm{S} / \mathrm{G}_{2}$ transition was higher than that of cells in the $\mathrm{G}_{1} / \mathrm{S}$ transition (Fig. 4, lower panel). This indicated that Hep-2 cells were obstructed in the $\mathrm{S} / \mathrm{G}_{2}$ transition following siRNA treatment. 

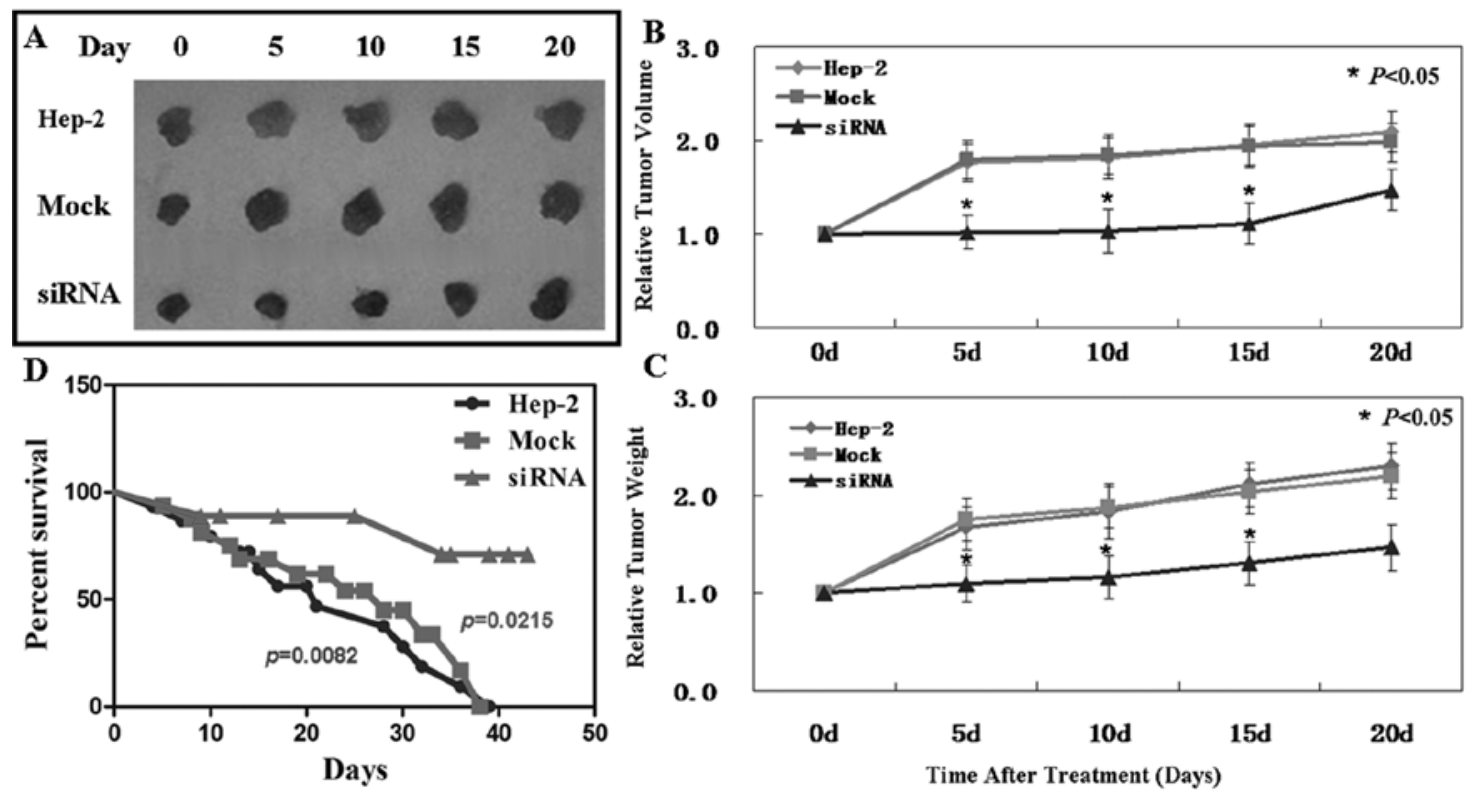

Figure 5. Downregulation of Pak4 suppressed tumor growth in the xenograft mouse model. (A) Subcutaneous tumors derived from Hep-2 cells were treated with PBS (Hep-2), control siRNA (Mock), or Pak4-siRNA (siRNA). (B and C) The downregulation of Pak4 expression inhibited tumor growth. Mice were sacrificed at the indicated time-points after treatment with PBS, control siRNA, or Pak4-siRNA. Tumor volume and tumor weight were measured. (D) The survival rate of mice in the Pak4 siRNA group was significantly higher than mice in the control groups (p<0.05). Each experiment was performed in triplicate.

In vivo anti-tumor effect of Pak4-siRNA on laryngeal carcinoma xenografts. The anti-tumor effect of Pak4-siRNA was analyzed in vivo using Hep-2 laryngeal carcinoma subcutaneous xenografts. When the established tumors reached 5-7 $\mathrm{mm}$ in diameter, siRNA was injected into the tumors. Treatment with siRNA was performed by mixing the plasmid DNA $(20 \mu \mathrm{g})$ with Lipofectamine $2000(30 \mu \mathrm{l})$ and PBS in a total volume of $100 \mu \mathrm{l}$ per mouse and injecting the mice intratumorally twice every 3 days. As is shown in Fig. 5B, on day 15 , the tumor volume of the control group was $623.1 \pm 43.3 \mathrm{~mm}^{3}$, that of the mock-treated group was $637.4 \pm 48.6 \mathrm{~mm}^{3}$ and that of the siRNA-treated group was $324.6 \pm 32.7 \mathrm{~mm}^{3}$. These results demonstrate the suppressive effect of Pak4-siRNA on tumor growth. Moreover, Pak4-siRNA showed the same effects on tumor weight (Fig. 5C). However, on day 20, tumor volume and weight showed no significant differences between the 3 groups. Subsequently, the effectiveness of siRNA on the xenografts gradually diminished after 15 days.

A significantly improved survival rate was observed in the mice treated with siRNA (Fig. 5D). The mice began to die first in the siRNA-treated group on day 9, and then in the control and mock-treated groups on days 3 and 4, respectively. At the end of this experiment, there were 10 out of 15 mice left alive in the siRNA group. However, all mice died in the other 2 groups.

Downregulation of Pak4 causes $S$ phase arrest and is associated with the upregulation of p53. To identify the mechanism of the cell cycle arrest induced by Pak4, immunohistochemical staining was performed to detect changes in cell cycle-related proteins. The results demonstrated that the downregulation of Pak4 induced a significant decrease in the levels of cyclin A (Fig. 6A). Decreased levels of CDK2 were also detected (Fig. 6A). In further experiments, we found that p53 was acti- vated following the downregulation of Pak4 (Fig. 6A). These results indicate that the cell cycle arrest is dependent on p53 function in these cells.

Downregulation of Pak4-induced arrest at the $S / G_{2}$ transition depends on both Chk1 and Chk2. The protein kinases, ATM and Chk1/Chk2, are major components of the mechanisms that oversee the control of DNA replication and genomic integrity (18). In our study, we found that the levels of Chk1 and Chk2 were not altered in the siRNA-treated group compared with the control and mock-treated groups (Fig. 6B). However, the levels of P-Chk1 and P-Chk2 were significantly increased in the siRNA-treated group (Fig. 6B). To examine the hypothesis that the ATM/Chk1/Chk2-p53 pathway is activated by the downregulation of Pak4 in the siRNA-treated group, we detected the levels of ATM, the upstream protein of Chk1 and Chk2. We found that ATM levels also increased (Fig. 6B). These data are consistent with the results of the cell cycle arrest at the $\mathrm{S} / \mathrm{G}_{2}$ transition.

\section{Discussion}

Pak4 was originally identified as a protein which binds strongly to $\mathrm{Cdc} 42$ and mediates Cdc42-induced cytoskeletal organization and cell shape (9). Gnesutta et al found that Pak4 regulates cell growth and survival (19). There is increasing evidence that Pak4 is overexpressed in human tumors and cancer cell lines (10-12). Consistent with previous studies, in this study, we demonstrate that the serine/threonine kinase, Pak4, is overexpressed in Hep-2 cells. In order to determine the role of Pak4 in Hep-2 cells, we established Pak4-knockdown cell lines by using siRNA. We also confirmed that the downregulation of Pak4 inhibits proliferation and induce $S$ phase arrest in Hep-2 cells. Similarly, Pak4 knockdown displayed anti-tumor 
A

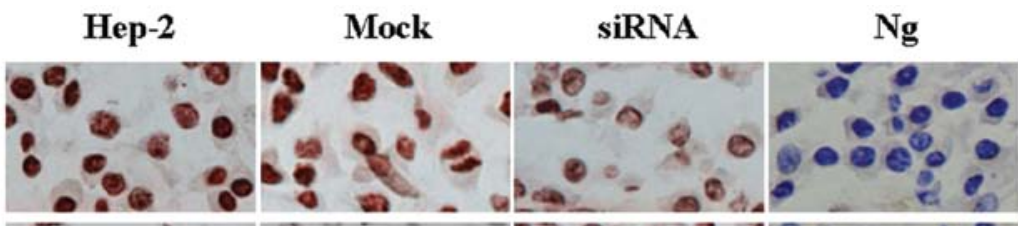

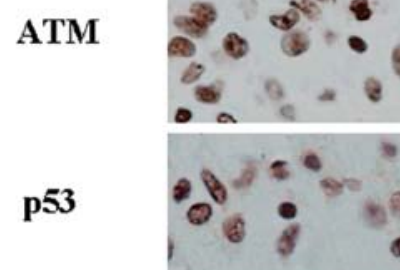

Pak

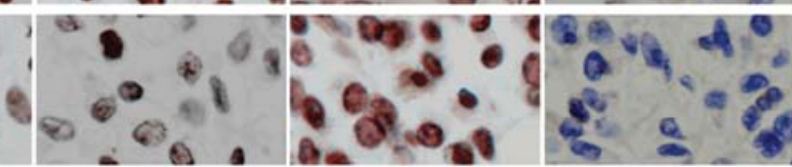

Cyclin A

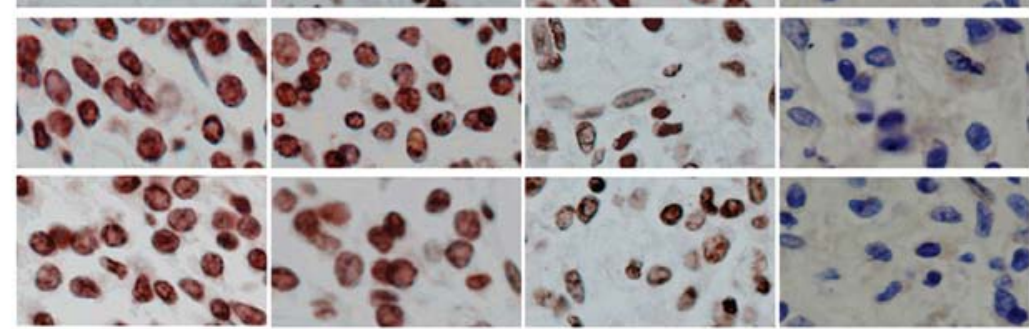

B

CDK2
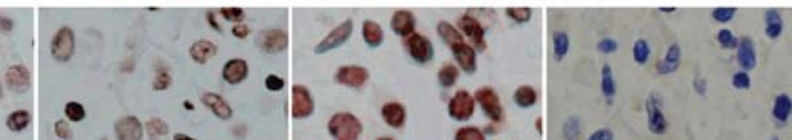

p-Chk1 ${ }^{\mathrm{s} 345}$

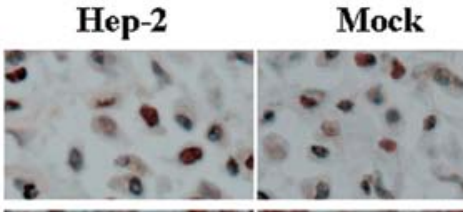

siRNA

$\mathrm{Ng}$

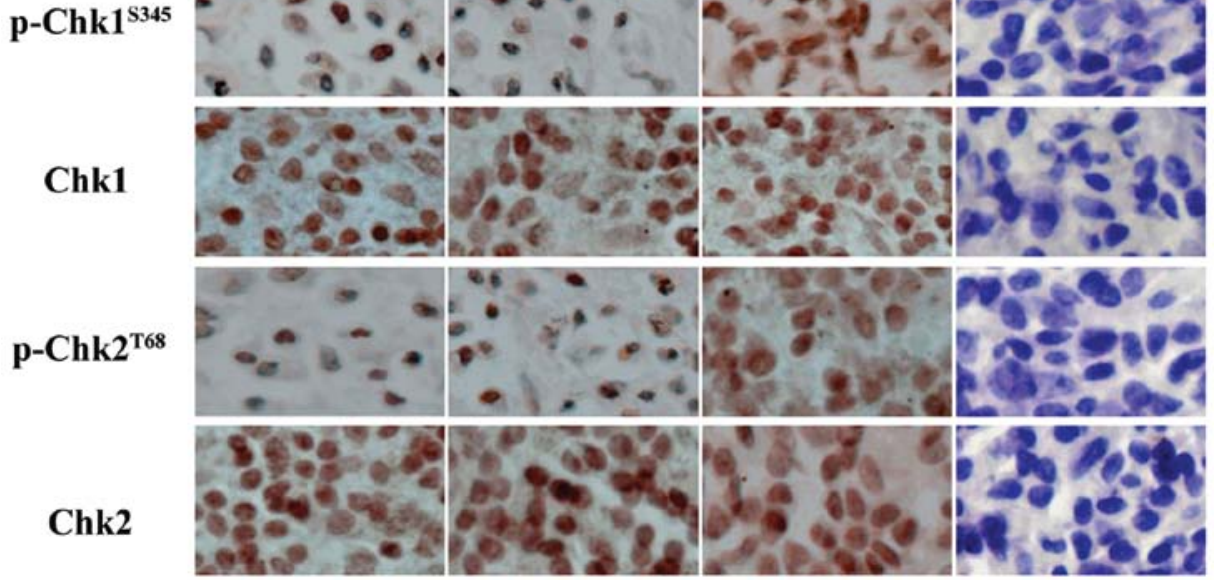

Figure 6. Immunohistochemical staining analysis of cell cycle-related proteins. (A) Cyclin A and its kinase CDK2 were detected by immunohistochemical analysis. The results showed that the levels of Cyclin A and CDK2, the S phase-related proteins, were decreased. (A and B) The upstream proteins, ATM and p53 were overexpressed. p-Chk1 and p-Chk2 were increased following siRNA treatment. No significant changes were observed in the levels of Chk1 and Chk2.

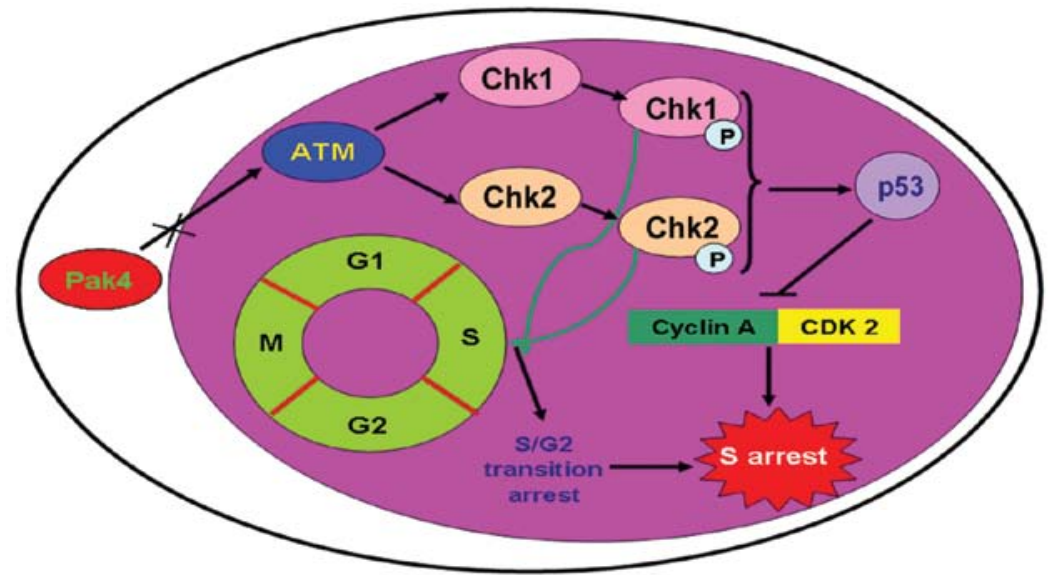

Figure 7. The proposed model of the signaling pathway of Pak4, which explains the mechanism of the of Pak4-induced $\mathrm{S} / \mathrm{G}_{2}$ arrest in Hep-2 laryngeal carcinoma cells. 
activities in laryngeal carcinoma xenografts. In further experiments, by using CldU and IdU double staining, we found that Pak4 knockdown obstructed $\mathrm{S} / \mathrm{G}_{2}$ transition.

Through the course of detecting the mechanism of S arrest, we found that the levels of cyclin A and CDK2 were decreased. Beamish et al (20) also found that the inhibition of cyclin A and CDK2 resulted in mitotic cell arrest with the activation of the spindle assembly checkpoint. We confirmed that p53, the inhibitor of cyclin A, was activated by Pak4 knockdown. This is the cause of the inhibition of cyclin A.

Indeed, the most significant results of our study was that Pak4 knockdown obstructed $\mathrm{S} / \mathrm{G}_{2}$ transition. Chk2 plays important roles in the DNA damage response, the signaling of the ATM/ Chk2/p53 pathway and in cell cycle checkpoints including the $\mathrm{S}$ checkpoint $(21,22)$. The response typically leads to the activation of p53, predominantly through ATM and Chk2 $(23,24)$. The outcome of $\mathrm{p} 53$ activation ranges from cell cycle arrest and DNA repair to apoptosis $(25,26)$. Consistent with previous studies, we found that the ATM/Chk2/p53 pathway was activated by Pak4 knockdown. The expression of ATM was higher in the treated compared to the untreated cells. Accompanied with the upregulation of ATM, p-Chk2 was also increased. Of note, in our study, Chk1 was also activated by Pak4 knockdown. Chk1 plays an important role in DNA repair and is essential for the maintenance of genomic stability $(27,28)$. In previous studies, Ahmed et al (29) found that the small molecule, reactivation of p53 and induction of tumor cell apoptosis (RITA), activated the canonical ATM/ATR DNA damage response pathway that leads to the activation of Chk1 and Chk2 phosphorylation. In our study, we also confirmed that the phosphoryltion of Chk1 and Chk2 was increased accompanied with the activation of ATM.

In summary, we confirmed the anti-tumor effects of the downregulation of Pak4 on Hep-2 laryngeal carcinoma cells in all our experiments. We found that the downregulation of Pak4 activated the ATM/Chk1/2/p53 signaling pathway in laryngeal carcinoma cells. After the signaling pathway was activated, apoptosis was induced by activated caspase- 3 and caspase- 9 . We also found that activation of the ATM/Chk1/2/p53 pathway promoted $\mathrm{S} / \mathrm{G}_{2}$ transition arrest (Fig. 7). The data presented in our study maybe provide a novel insight into laryngeal carcinoma treatment.

\section{Acknowledgements}

We thank Dr Dong-ying Wu for his valuable comments and excellent technical assistance.

\section{References}

1. Van Aelst L and D'Souza-Schorey C: Rho GTPases and signaling networks. Genes Dev 11: 2295-2322, 1997.

2. Bagrodia S, Dérijard B, Davis RJ and Cerione RA: Cdc42 and PAK-mediated signaling leads to Jun kinase and p38 mitogen-activated protein kinase activation. J Biol Chem 270: 27995-27998, 1995.

3. Dutartre H, Davoust J, Gorvel JP and Chavrier P: Cytokinesis arrest and redistribution of actin-cytoskeleton regulatory components in cells expressing the Rho GTPase CDC42Hs. J Cell Sci 109: 367-377, 1996.
4. Coso OA, Chiariello M, Yu JC, et al: The small GTP-binding proteins Rac1 and Cdc42 regulate the activity of the JNK/SAPK signaling pathway. Cell 81: 1137-1146, 1995.

5. Minden A, Lin A, Claret FX, et al: Selective activation of the JNK signaling cascade and c-Jun transcriptional activity by the small GTPases Rac and Cdc42Hs. Cell 81: 1147-1157, 1995.

6. Brown JL, Stowers L, Baer M, et al: Human Ste20 homologue hPAK1 links GTPases to the JNK MAP kinase pathway. Curr Biol 6: 598-605, 1996.

7. Kumar R, Gururaj AE and Barnes CJ: p21-activated kinases in cancer. Nat Rev Cancer 6: 459-471, 2006.

8. Arias-Romero LE and Chernoff J: A tale of two Paks. Biol Cell 100: 97-108, 2008.

9. Abo A, Qu J, Cammarano MS, et al: PAK4, a novel effector for $\mathrm{Cdc} 42 \mathrm{Hs}$, is implicated in the reorganization of the actin cytoskeleton and in the formation of filopodia. EMBO J 17: 6527-6540, 1998.

10. Callow MG, Clairvoyant F, Zhu S, et al: Requirement for PAK4 in the anchorage-independent growth of human cancer cell lines. J Biol Chem 277: 550-558, 2002.

11. Fang MZ, Jin Z, Wang Y, et al: Promoter hypermethylation and inactivation of $\mathrm{O}_{6}$-methylguanine-DNA methyltransferase in esophageal squamous cell carcinomas and its reactivation in cell lines. Int J Oncol 26: 615-622, 2005.

12. Thompson HJ and Singh M: Rat models of premalignant breast disease. J Mammary Gland Biol Neoplasia 5: 409-420, 2000.

13. Jemal A, Bray F, Center MM, et al: Global cancer statistics. CA Cancer J Clin 61: 69-90, 2011.

14. Che XH, Chen H, Xu ZM, et al: 14-3-3epsilon contributes to tumour suppression in laryngeal carcinoma by affecting apoptosis and invasion. BMC Cancer 10: 306, 2010.

15. Alessandri G, Filippeschi S, Sinibaldi P, et al: Influence of gangliosides on primary and metastatic neoplastic growth in human and murine cells. Cancer Res 47: 4243-4247, 1987.

16. Dover R and Patel K: Improved methodology for detecting bromodeoxyuridine in cultured cells and tissue sections by immunocytochemistry. Histochemistry 102: 383-387, 1994.

17. Bakker PJ, Stap J, Tukker CJ, et al: An indirect immunofluorescence double staining procedure for the simultaneous flow cytometric measurement of iodo- and chlorodeoxyuridine incorporated into DNA. Cytometry 12: 366-372, 1991.

18. Nojima H: Protein kinases that regulate chromosome stability and their downstream targets. Genome Dyn 1: 131-148, 2006.

19. Gnesutta N, Qu J and Minden A: The serine/threonine kinase PAK4 prevents caspase activation and protects cells from apoptosis. J Biol Chem 276: 14414-14419, 2001.

20. Beamish H, de Boer L, Giles N, et al: Cyclin A/cdk2 regulates adenomatous polyposis coli-dependent mitotic spindle anchoring. J Biol Chem 284: 29015-29023, 2009.

21. Stracker TH, Usui T, Petrini JH: Taking the time to make important decisions: the checkpoint effector kinases Chk1 and Chk2 and the DNA damage response. DNA Repair (Amst) 8: 1047-1054, 2009.

22. Löbrich $M$ and Jeggo PA: The impact of a negligent G2/M checkpoint on genomic instability and cancer induction. Nat Rev Cancer 7: 861-869, 2007.

23. Karlseder J, Broccoli D, Dai Y, et al: p53- and ATM-dependent apoptosis induced by telomeres lacking TRF2. Science 283: 1321-1325, 1999.

24. Antoni L, Sodha N, Collins I and Garrett MD: CHK2 kinase: cancer susceptibility and cancer therapy - two sides of the same coin? Nat Rev Cancer 7: 925-936, 2007.

25. Wells BS, Yoshida E and Johnston LA: Compensatory proliferation in Drosophila imaginal discs requires Dronc-dependent p53 activity. Curr Biol 16: 1606-1615, 2006.

26. Shrivastav M, De Haro LP and Nickoloff JA: Regulation of DNA double-strand break repair pathway choice. Cell Res 18: 134-147, 2008.

27. Niida H, Murata K, Shimada M, et al: Cooperative functions of Chk1 and Chk2 reduce tumour susceptibility in vivo. EMBO J 29: 3558-3570, 2010.

28. Paulsen RD and Cimprich KA: The ATR pathway: fine-tuning the fork. DNA Repair (Amst) 6: 953-966, 2007.

29. Ahmed A, Yang J, Maya-Mendoza A, et al: Pharmacological activation of a novel p53-dependent S-phase checkpoint involving CHK-1. Cell Death Dis 2: e160, 2011. 